\title{
A Resonant Micromachined Electrostatic Charge Sensor
}

\author{
Yong Zhu, Joshua E.-Y. Lee, Student Member, IEEE, and Ashwin A. Seshia, Member, IEEE
}

\begin{abstract}
A micromachined electrometer, based on the concept of a variable capacitor, has been designed, modeled, fabricated, and tested. The device presented in this paper functions as a modulated variable capacitor, wherein a dc charge to be measured is up-modulated and converted to an ac voltage output, thus improving the signal-to-noise ratio. The device was fabricated in a commercial standard SOI micromachining process without the need for any additional processing steps. The electrometer was tested in both air and vacuum at room temperature. In air, it has a charge-to-voltage conversion gain of $2.06 \mathrm{nV} / \mathrm{e}$, and a measured charge noise floor of $52.4 \mathrm{e} / \mathrm{rtHz}$. To reduce the effects of input leakage current, an electrically isolated capacitor has been introduced between the variable capacitor and input to sensor electronics. Methods to improve the sensitivity and resolution are suggested while the long-term stability of these sensors is modeled and discussed.
\end{abstract}

Index Terms-Electrostatic charge sensor, micromechanical resonator, transcapacitance amplifier (TCA), variable capacitor, vibrating reed electrometer.

\section{INTRODUCTION}

$\mathbf{E}$ LECTROMETERS are instruments used to measure charge and have found widespread applications in nuclear research [1], space exploration [2], and mass spectrometry for chemical and biological analyses [3]. At present, cryogenically cooled single electron transistors [4] are considered to be the best electrometers, with reported noise levels of $10^{-5} \mathrm{e} / \sqrt{\mathrm{Hz}}$. Subelectron charge resolution has also been achieved using nanomechanical torsional beam structures, in which the measured input charge induces a shift in the natural frequency of the torsional beam [5]. More recently, other detection techniques using clamped-clamped beam resonators with similar reported noise levels have also been demonstrated [6]. Although capable of very high sensitivity, these techniques require extremely low temperatures $(<4.2 \mathrm{~K})$ to achieve subelectron resolution or better. Work on small scale electrometers functioning under room temperature and ambient conditions has been limited. A technique based on the macroscale size vibrating reed capacitor [7], [8] implemented as a micromechanical device shows great promise [9], [10], wherein a charge resolution of approximately

Manuscript received October 5, 2007; revised January 28, 2008; accepted February 4, 2008. This work was supported by the U.S. Army Soldier Systems Centre. The associate editor coordinating the review of this paper and approving it for publication was Dr. Usha Varshney.

The authors are with the Department of Engineering, University of Cambridge, Cambridge CB2 1PZ, U.K. (e-mail: yongyan.zhu@googlemail.com; eyj12@cam.ac.uk; aas41@cam.ac.uk).

Color versions of one or more of the figures in this paper are available online at http://ieeexplore.ieee.org.

Digital Object Identifier 10.1109/JSEN.2008.923597

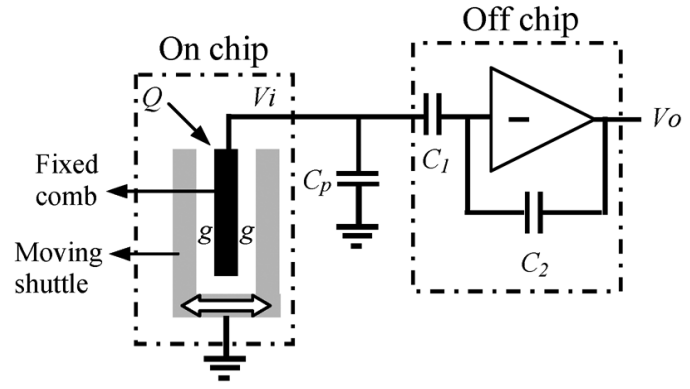

Fig. 1. Schematic of micromachined electrometer including variable capacitor and transcapacitance amplifier.

$51 \mathrm{e} / \mathrm{rtHz}$ was demonstrated at room temperature and ambient pressure. This concept uses a balanced comb resonator that modulates a dc charge input to an ac voltage output with a primary frequency component that is twice the modulating frequency. Detection is carried out at this second harmonic frequency, which substantially reduces the effects of charge feedthrough and $1 / f$ noise from the sensor electronics.

We have reported a MEMS electrometer with a measured charge noise floor of $6 \mathrm{e} / \mathrm{rtHz}$ [11]. In order to achieve high resolution, a low-noise JFET was implemented in the input buffer of the sensor electronics. Although the input bias current of JFETs are typically much smaller relative to bipolar transistors, this quiescent charge leakage at the input node is substantial when measuring precise amounts of charge. This adds to the difficulty in quantifying small increments of charge, and renders the electrometer inoperable in vacuum due to suspected electrostatic pull-in instability that is currently being investigated further.

In this paper, we develop a new setup for the subtle charge measurement, based on the micromechanical vibrating reed electrometer. Here, the induced charge is applied to a capacitor, and not active electrical devices. This leads to extremely low leakage charges, which is a major advantage compared to the electrometers presented in [9]-[11]. In comparison to an input JFET readout, the proposed setup based on a transcapacitance amplifier interface may be readily implemented to realize a fully integrated CMOS-MEMS high-resolution electrometer.

\section{SENSOR DESIGN}

The principle of the vibrating reed electrometer is schematically depicted in Fig. 1. The electrometer comprises a MEMS charge sensing element functioning as a variable capacitor that is coupled to interface sensor electronics for further voltage amplification. 


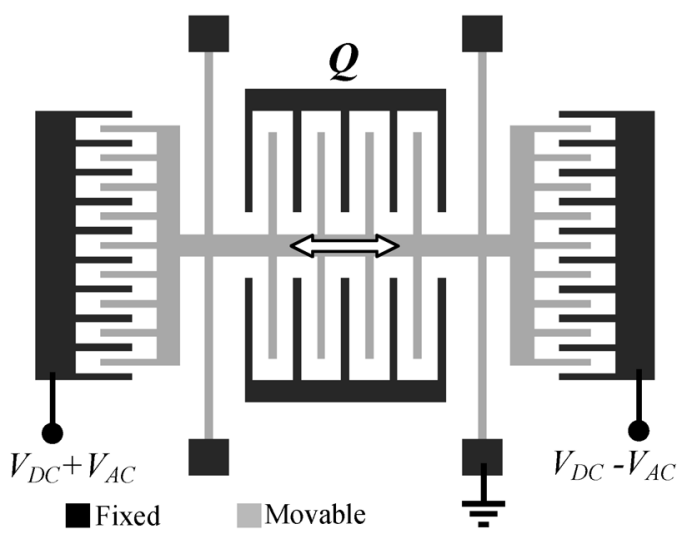

Fig. 2. Schematic of micromachined variable capacitor.

A given input charge $Q$ loaded at the input node produces a converted ac voltage signal, as illustrated by the following relation:

$$
V_{i}(t)=Q /\left(C_{V}(t)+C_{P}\right) .
$$

Depending on the geometry and topology of the variable capacitor $C_{V}(t)$, a corresponding associated converted voltage is obtained with a characteristic frequency spectrum.

The charge to be measured is input to a fixed electrode that forms a parallel-plate variable capacitor. The basic variable capacitor structure, shown in Fig. 2, is constructed from an array of electrodes extending from a movable shuttle suspended by flexural beams that are anchored at one end and guided by the shuttle at the other end, interjected by the fixed electrodes to form a parallel array of capacitors. The variable capacitor is harmonically driven using lateral comb drive actuators, which provide a drive force that is nominally independent of the displacement. This driving force is a function of only the drive voltage and actuator geometry.

In this configuration, the movable electrode is sandwiched between fixed electrodes on each side, with equal gaps on either side. For such a structure, the time-modulated capacitance is given by

$$
C_{V}(t)=C_{0}\left[\frac{g}{g+x(t)}+\frac{g}{g-x(t)}\right]
$$

where $C_{0}$ is the nominal value of the variable capacitor, and $g$ is the gap between the sense electrodes of the variable capacitor.

Since comb drive actuation is used, the motion of the resonator may be assumed to be closed to harmonic, and thus the lateral motion may be expressed as

$$
x(t)=\hat{x} \cos \omega t
$$

where $\hat{x}$ is the amplitude of the shuttle displacement, and $\omega$ denotes the angular frequency of modulation.
TABLE I

VARIable CAPACITOR Design PARAMETER VALUES

\begin{tabular}{cc}
\hline \hline DESIGN PARAMETER & VALUE \\
\hline Structure thickness $(\mu \mathrm{m})$ & 25 \\
Mass of movable structure $(\mu \mathrm{g})$ & 100 \\
Suspension beam width $(\mu \mathrm{m})$ & 4 \\
Suspension beam length $(\mu \mathrm{m})$ & 400 \\
Total spring constant $(\mathrm{N} / \mathrm{m})$ & 33 \\
Undamped natural frequency $(\mathrm{kHz})$ & 2.9 \\
Sense capacitor gap $(\mu \mathrm{m})$ & 5 \\
Number of sense combs per side & 145 \\
Sense capacitance per side $(\mathrm{pF})$ & 1.28 \\
Drive capacitor gap $(\mu \mathrm{m})$ & 3 \\
Number of drive combs per side & 684 \\
dC/dx of drive combs $(\mathrm{F} / \mathrm{m})$ & $101 \mathrm{E}-9$ \\
\hline \hline
\end{tabular}

Combining (1), (2), and (3), and ignoring frequency components beyond the second harmonic, the voltage induced on the variable capacitor $C_{V}$ can be written as

$$
\begin{aligned}
V_{i}(t)=Q\left[\frac{1}{C_{p}+C_{0}}-\frac{\hat{x}^{2}}{g^{2}}\right. & \frac{C_{0}}{2\left(C_{0}+C_{p}\right)^{2}} \\
& \left.-\frac{\hat{x}^{2}}{g^{2}} \frac{C_{0}}{2\left(C_{0}+C_{p}\right)^{2}} \cos 2 \omega t\right]
\end{aligned}
$$

where $C_{p}$ is the total parasitic capacitance to ground at the input.

As illustrated in (4), the charge is modulated from dc to a voltage output with frequency components at dc and at twice the modulation frequency of the variable capacitor, a unique characteristic of the balanced comb structure. Detection is carried out at twice the modulation frequency to suppress electrical drive feedthrough interference. The RMS magnitude of this second harmonic component of the output voltage varies linearly with the input charge and is given by the expression

$$
\frac{d V_{i}}{d Q}=\frac{C_{V}}{2 \sqrt{2}\left(C_{V}+C_{P}\right)^{2}}\left(\frac{\hat{x}}{g}\right)^{2} .
$$

This slope which relates input charge to output voltage defines the sensitivity of the variable capacitor, a figure of merit whose optimization improves instrument resolution.

Note that (4) and (5) are only for the variable capacitor itself, excluding the gain of interface electronics. A transcapacitance amplifier (TCA) topology was chosen to reduce the leakage charge from interface electronics, which will be discussed in greater detail subsequently in Section IV.

The variable capacitor was designed for resonance at around $2.9 \mathrm{kHz}$, verified with simulations in IntelliSuite. Table I summarizes the design parameters for the MEMS modulated variable capacitor, while an SEM of a fabricated device is shown in Fig. 3.

\section{SENSOR FABRICATION}

The devices were fabricated in a commercial foundry silicon-on-insulator (SOI) MEMS technology available from 


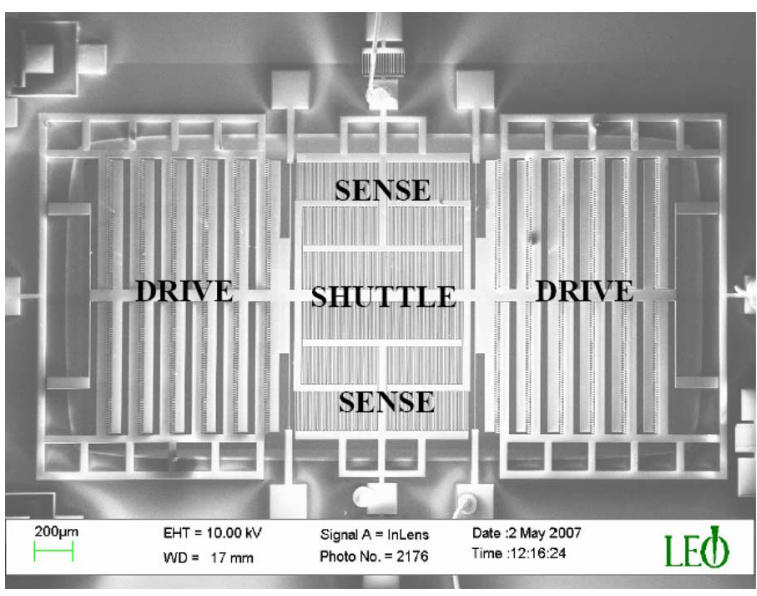

Fig. 3. SEM of the micromachined variable capacitor.
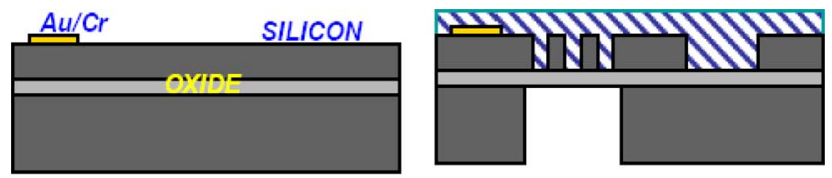

1) Metallization

4) Backside trench DRIE
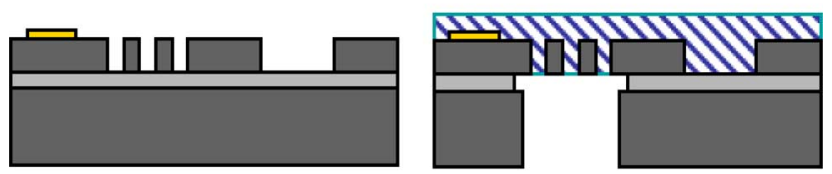

2) Front side DRIE

5) Backside HF wet etch

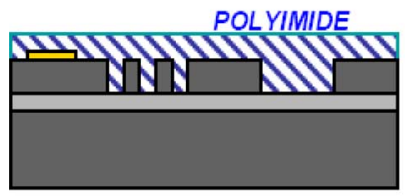

3) Front side protection

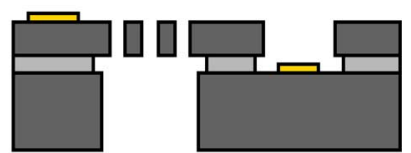

6) Remove polyimide

Fig. 4. SOI MEMS fabrication processes from MEMSCAP

MEMSCAP [12]. Fig. 4 provides an illustrated summary of this process.

1) Surface metal pads are patterned on a highly doped n-type $25 \mu \mathrm{m}$ silicon device layer to allow for ohmic contact.

2) Deep reactive ion etch (DRIE) from the front side of the wafer to define both the anchored and movable features of the structure.

3) Protective polyimide layer is applied to the front side.

4) Deep trench underneath the movable structures is created by etching through the substrate using DRIE.

5) Exposed buried oxide is removed using a wet HF etch.

6) Polyimide coat on the front side is removed by oxygen plasma, thereby allowing the movable structure to be fully released. Then a large contact metal pad is patterned on the substrate for electrical contact.

A backside release process has the advantage of reducing the input parasitic capacitance. Since the underlying substrate beneath the suspended structures of the modulated capacitor is completely removed, input parasitic contributions from the

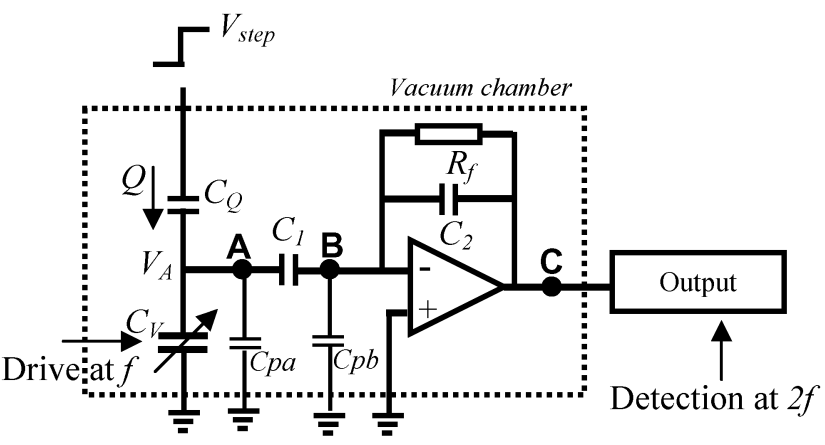

Fig. 5. Schematic measurement setup of the electrometer.

TABLE II

ELECTROMETER SPECIFICATION

\begin{tabular}{cc}
\hline \hline PARAMETER & VALUE \\
\hline Test capacitor $\mathrm{C}_{\mathrm{Q}}$ & $0.236 \mathrm{pF}$ \\
Isolation capacitor $\mathrm{C}_{1}$ & $2 \mathrm{pF}$ \\
Feedback capacitor $\mathrm{C}_{2}$ & $1.2 \mathrm{pF}$ \\
Variable capacitor $\mathrm{C}_{\mathrm{V}}$ & $1.28 \mathrm{pF}$ \\
Parasitic capacitance $\mathrm{C}_{\mathrm{pa}}, \mathrm{C}_{\mathrm{pb}}$ & $2 \mathrm{pF}$ \\
Opamp noise & $6 \mathrm{nV} / \mathrm{rtHz} @ 10 \mathrm{kHz}$ \\
Feedback resistor $\mathrm{R}_{\mathrm{f}}$ & $50 \mathrm{G} \Omega$ \\
Drive frequency $f$ & $3.2 \mathrm{kHz}$ \\
\hline \hline
\end{tabular}

static capacitance arising from between the suspended sense electrodes and grounded substrate is consequently greatly reduced. An identical structure suspended over a substrate by a height of $1 \mu \mathrm{m}$ adds a considerable extra $2 \mathrm{pF}$ to the input parasitic. As already mentioned, optimization of the charge to voltage conversion gain improves charge resolution. Equation (5) shows that the sensitivity is largely dependent on the ratios between the sense and parasitic capacitances, such that reducing the parasitics allows for substantial improvement in electrometer charge resolution. In short, the fabrication process of releasing structures from the backside by trench etching through the substrate is favorable for enhancing the device performance in this case.

\section{MEASurement SetuP}

In most applications, one can ignore the gate current of an FET device. This is not the case in high precision electrometry due to the extremely miniscule amounts of charge that are measured. In order to reduce the current in the gate leakage path, a fixed isolation capacitor is introduced between the variable capacitor and the input of the sensor electronics. Fig. 5 shows the circuit schematic for the electrometer with isolation capacitor $C_{1}$ between the variable capacitor and the input of the TCA. The detailed device parameters are listed in Table II.

In this topology, charge is injected through a capacitor $C_{Q}$ by applying a voltage step of $V_{\text {step }}$. The charge accumulates on the variable capacitor $C_{v}$, implemented by a micromachined resonator, which is driven to vibrate at frequency $f$. Detection at the second harmonic has the benefit of rejecting substantial signal feedthrough from the electrodes used to drive the movable shuttle, which could potentially obscure small signal levels at the sense input, thus limiting the performance of the electrometer. The extent of drive feedthrough rejection at the second 
harmonic relative to detection at the modulation frequency depends on the second harmonic distortion of the drive signal, and is usually of the order of $-60 \mathrm{~dB}$.

A capacitor $C_{1}$ electrically isolates the charge node $A$ from the active electronic device (low noise opamp), thus dramatically reducing the leakage current to node $A$, in comparison to the case where an FET buffer topology is used [9]-[11]. The gate leakage current flows into the opamp output node through a feedback resistor $R_{f}(50 \mathrm{G} \Omega$ ), which fixes the dc bias of node $\mathrm{B}$ and $\mathrm{C}$ close to $0 \mathrm{~V}$, since the bias current of the selected opamp is typically only $1 \mathrm{pA}$. An initial voltage offset at the input induces a corresponding offset charge across the variable capacitor. Hence, a very low voltage offset at the input is beneficial for reducing such an initial offset in the output. The ac voltage signal at $A$ is then amplified by the TCA with a gain set by the ratio $C_{1} / C_{2}$. The value of $R_{f}$ is chosen to be in the range of $\mathrm{G} \Omega \mathrm{s}$ to allow for a cutoff frequency much lower than the operating frequency of the resonator. A large feedback resistor is also advantageous for limiting the input referred noise of the amplifier. These design issues are further discussed in Section V.

To reduce the ambient leakage current and noise, the variable capacitor, isolation capacitor, TCA, and drive circuits were placed inside a grounded custom-made vacuum chamber.

\section{ANALYSIS}

\section{A. Sensitivity}

Generally, the electrometer sensitivity is the product of the charge to voltage gain of variable capacitor and TCA voltage gain. The parasitic capacitor includes all the capacitance to ac ground from node $\mathrm{A}$, which includes $C_{1}, C_{p a}$, and $C_{Q} . C_{1}$ is included in the lumped parasitic capacitor because it is connected to a virtual ground of the opamp, thereby sharing some of the charge at the input. If the impedance of $R_{f}$ is much larger than $C_{2}$ at the working frequency $2 f$, the gain of the TCA is $C_{1} / C_{2}$. Combining the (5), the sensitivity is given by

$$
\begin{aligned}
S_{e} & =\frac{d V_{i}}{d Q}\left(\frac{C_{1}}{C_{2}}\right) \\
& =\frac{C_{V}}{2 \sqrt{2}\left(C_{V}+C_{1}+C_{p a}+C_{Q}\right)^{2}}\left(\frac{\hat{x}}{g}\right)^{2}\left(\frac{C_{1}}{C_{2}}\right) .
\end{aligned}
$$

Equation (6) indicates that in order to increase the electrometer sensitivity, one could

1) increase the amplitude to variable capacitor gap ratio $(\widehat{x} / g)$

2) reduce parasitic capacitance from node $\mathrm{A}$ to ground $\left(C_{p a}\right)$, which can be realized by an on-chip capacitor solution;

3 ) reduce $C_{2}$. However, $C_{2}$ cannot be too small since the uncertainty of the parasitic capacitance between B and C arising from both circuit board layout and the feedback resistor becomes increasingly significant as $C_{2}$ is reduced.

Fig. 6 shows that the electrometer sensitivity is at a maximum peak when the isolation capacitor is $3.5 \mathrm{pF}$. When $\mathrm{C}_{1}$ is larger than $3.5 \mathrm{pF}$, the decrease in the charge to voltage gain of the variable capacitor is greater than the increase in the TCA gain. This is due to the fact that above $3.5 \mathrm{pF}$, the proportion of charge

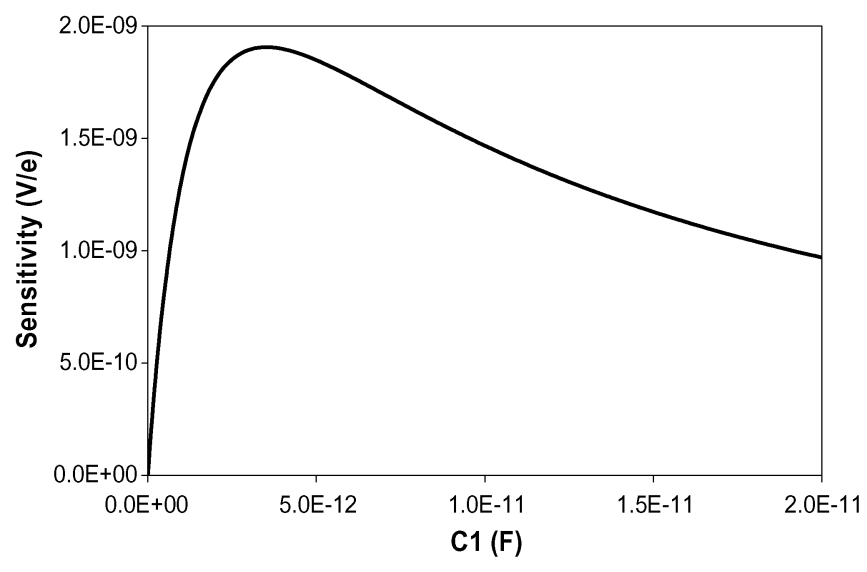

Fig. 6. Variation of electrometer sensitivity with different values of isolation capacitor $\mathrm{C}_{1}$, calculated using the parameter values in Table II.

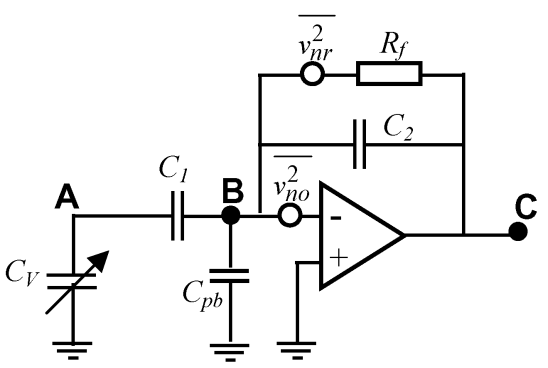

Fig. 7. Noise model of the electrometer.

shared by $\mathrm{C}_{1}$ is sufficiently large to offset further increases in the amplifier gain. The results were verified using a full-system simulation method based in SPICE that we have developed for the study of nonlinear variable capacitors [13].

\section{B. Noise}

The overall noise contribution includes Brownian noise from mechanical structures and Johnson noise from electronics. In the case of the variable capacitor electrometer, resolution is not limited by Brownian noise, unlike certain force-based sensing techniques that require very low temperatures for high-resolution detection [5]. For the variable capacitor based electrometer, the output voltage is proportional to the square of the ratio of the displacement amplitude of the micromechanical element to the capacitor gap $(\widehat{x} / g)$, both of which are in the order of a micron. The typical variance due to Brownian noise in the displacement of a structure of similar size scale as the variable capacitor is in the order of $1 \mathrm{pm}$. This is a variance on the order of 1 part per $10^{12}$, and hence Brownian noise does not place a limit to charge resolution. The resolution limit is dependent on the implementation of the interface electronics, in particular the electronic noise from the opamp and feedback resistor $R_{f}$, which is shown in Fig. 7.

To calculate the electronic noise contributed by the opamp, ground node $\mathrm{A}$ and omit the noise voltage of $R_{f}$, and the output noise contribution from the opamp (node $\mathrm{C}$ in Fig. 7) is given by

$$
\overline{v_{C_{-} o p}^{2}}=\overline{v_{n o}^{2}}\left[\left(C_{1}+C_{2}+C_{p b}\right) / C_{2}\right]^{2} .
$$




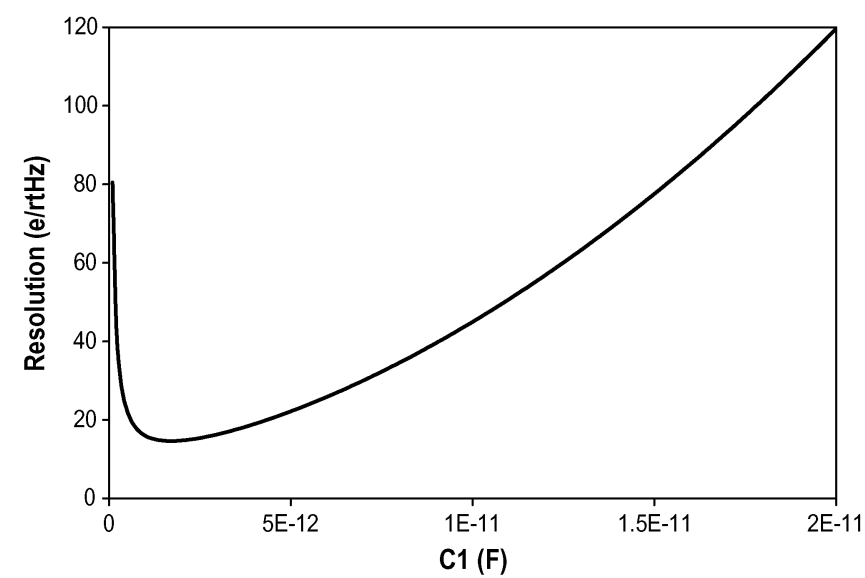

Fig. 8. Variation of electrometer resolution with different values of isolation capacitor $\mathrm{C}_{1}$, calculated using the parameter values in Table II.

Next, ground node A and omit the noise voltage of opamp, the output noise contributed by feedback resistance $R_{f}$ is

$$
\overline{v_{C_{-} R}^{2}}=4 k T / \omega^{2} R_{f} C_{2}^{2} .
$$

The charge resolution is finally given by

$$
\overline{Q_{n}}=\frac{\sqrt{\overline{v_{C_{-o p}}^{2}}+\overline{v_{C_{-} R}^{2}}}}{S_{e}} .
$$

Fig. 8 shows that the electrometer resolution is at a minimum peak when the isolation capacitor $C_{1}$ is $1.7 \mathrm{pF}$.

\section{EXPERIMENTAL RESULTS AND DISCUSSION}

The micromachined variable capacitor was packaged in a leadless chip carrier and wired bonded, and tested directly on a circuit board. The PCB containing variable capacitor, drive and sense circuits was tested in a custom-made vacuum chamber, which minimizes the ambient current leakage and noise. The electrometer was tested in both air and vacuum to investigate for possible differences.

\section{A. In Air}

The variable capacitor was driven differentially by applying antisymmetric voltage signals on each side of the drive electrodes. An ac voltage of $4 \mathrm{~V}$ peak, together with a dc bias of $30 \mathrm{~V}$, was applied at $3.2 \mathrm{kHz}$ to drive the device in air. The amplitude of the resonator was found to be roughly $2 / 3$ of variable capacitor gap, as observed under a microscope.

Known quantities of charge were then introduced on the anchored sense electrodes by adding known step voltages to a $0.236 \mathrm{pF}$ on-chip interdigitated air-gap silicon capacitor $C_{Q}$, which has been designed to reduce wiring parasitics present in our previous devices that used a surface mount capacitor on a PCB instead [14]. Increasing levels of voltages were applied to the on-chip capacitor using a Keithley 2400 source meter in steps of $0.05 \mathrm{~V}$ to load calibrated amounts of charge on the electrometer input, and the measured output was monitored on an Agilent 4396B analyzer in the spectrum analyzer mode.

Fig. 9 shows the output spectrum before and after $99 \mathrm{fC}$ charge is applied. Before inducing charge, there is a small peak

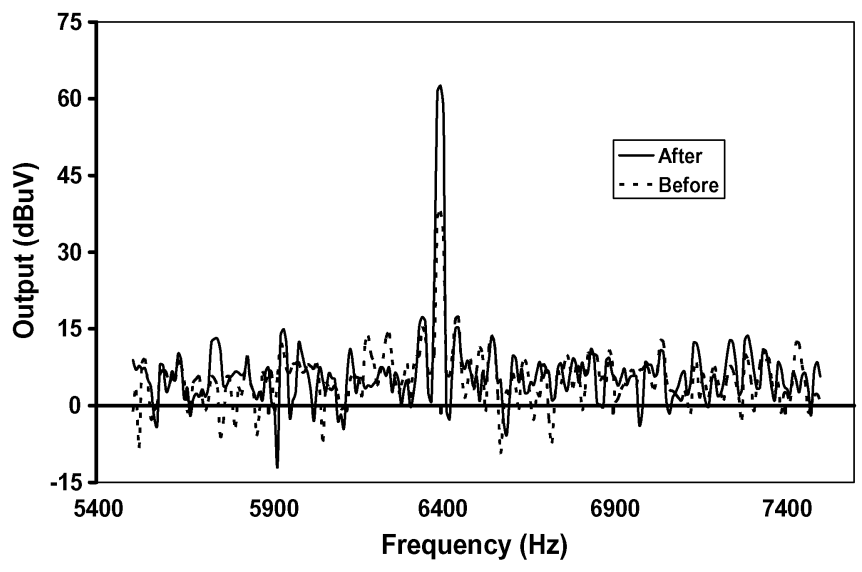

Fig. 9. Measured spectrum change of electrometer output when $99 \mathrm{fC}$ charge is introduced at the input.

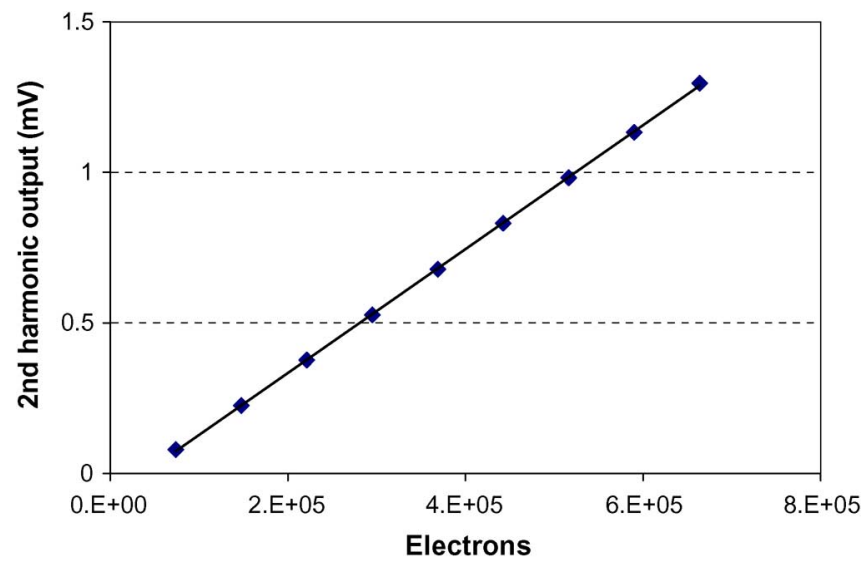

Fig. 10. Measured sensitivity of electrometer.

at $6.4 \mathrm{kHz}$, which is the second harmonic component, primarily due to quiescent charge at the input node. The source of this quiescent charge is very likely to arise from the subtle leakage current in the capacitor $C_{1}$. As seen from Fig. 9, upon introducing $99 \mathrm{fC}$ of charge through the on-chip test capacitor, the output peak changed from $37.9 \mathrm{dBuV}$ to $62.5 \mathrm{dBuV}$, verifying the functionality of the electrometer.

Fig. 10 shows the calibration curve obtained from these measures, which relates the input charge to the measured output voltage. The slope of the best-fit line to the calibration curve defines the sensitivity of the electrometer, which is $2.06 \mathrm{nV} / \mathrm{e}$ in this case. The noise floor of the output was measured with the input of the TCA grounded to find the input referred noise of the amplifier. This was found to be $108 \mathrm{nV} / \mathrm{rtHz}$. Using (9), this yields a measured equivalent charge noise floor of $52.4 \mathrm{e} / \mathrm{rtHz}$.

\section{B. In Vacuum}

The above results were obtained at room temperature and ambient pressure. The same experiments were conducted in vacuum in a purpose-built vacuum chamber that was sealed and pumped to 5.5 mTorr. However, since the structure was driven open-loop it was impossible to precisely lock-in to the resonance frequency in vacuum due to the much lower structural damping in vacuum. In addition, the vacuum chamber 
TABLE III

COMPARISON OF TEST RESULTS IN AIR AND VACUUM (5.5 mTorr)

\begin{tabular}{ccccc}
\hline \hline & $\mathrm{DC}(\mathrm{V})$ & $\mathrm{AC}(\mathrm{V})$ & $\begin{array}{c}\text { Sensitivity } \\
(\mathrm{nV} / \mathrm{e})\end{array}$ & $\begin{array}{c}\text { Resolution } \\
(\mathrm{e} / \mathrm{rtHz})\end{array}$ \\
\hline Air & 30 & 4 & 2.06 & 52.4 \\
Vacuum & 30 & 2.5 & 1.58 & 68.3 \\
\hline \hline
\end{tabular}

was not equipped with an optical window making it difficult to estimate the displacement of the moving electrode. As the damping in vacuum is much lower than that in air, the amplitude of motion of the moving electrodes will be larger in vacuum for the same drive force as applied in air. According to (6), one can roughly deduce the resonator motional amplitude to gap ratio by comparing the electrometer sensitivity in air and vacuum. The results outlined in Table III show that a $4 \mathrm{~V}$ ac drive voltage in air and a $2.5 \mathrm{~V}$ ac drive voltage in vacuum at the same drive frequency $(3.2 \mathrm{kHz})$ yield sensitivities which are approximately the same, indicating similar motional amplitudes. As the electronic noise is the same in either case, the resolutions are similar in air and in vacuum based on (9). The resolution in vacuum is slightly poorer than that in air due to lower sensitivity. One could in theory apply a larger ac voltage to increase the sensitivity, though in doing so one runs the risk of stiction between the movable and anchored combs resulting from excessively large displacements.

\section{Stability}

The high sensitivity of the device acts as a double-edged sword. Since the electrometer is designed to sense extremely subtle amounts of charge, the device is consequently also highly sensitive to a number of undesired charge inducing phenomena. Some sources of uncertainty include photoemission, ionizing radiation, and parasitic current leakage paths. After starting up the electrometer, one should wait several minutes to allow the readings to stabilize. This equilibrium is unbalanced each time charge is added through the test capacitor, which in turn requires some time to elapse before equilibrium is eventually restored. To investigate the transient behavior of the electrometer, we applied a step voltage from $-5 \mathrm{~V}$ to $4 \mathrm{~V}$ to the test capacitor $C_{Q}$ in air and in vacuum respectively and observed how the output responds, in order to provide insight into some of the fundamental physics.

Fig. 11 shows the measured charge varies with time after an initial instantaneous rise in the output upon applying a voltage step from $-5 \mathrm{~V}$ to $4 \mathrm{~V}$ to the $0.236 \mathrm{pF}$ test capacitor. For clarity of presentation, both curves in Fig. 11 have been initialized to start at the same output value at $0 \mathrm{~min}$ regardless of the value of this initial rise in the output. The figure shows that after $0 \mathrm{~min}$, both curves continue to rise steadily and plateau off at around $8 \mathrm{~min}$. This peak is followed by a gradual decrease $(8-40 \mathrm{~min})$. It may be observed that the peak value and the increasing rate in air are much larger than those in vacuum. Fig. 12 provides a circuit schematic of a model to explain this behavior.

In an idealized setting, a capacitor can be seen as an open circuit, because the dielectric resistance is very high, such that we assume there is always no active dc current through it. However, this assumption is not valid for high precision electrometry due

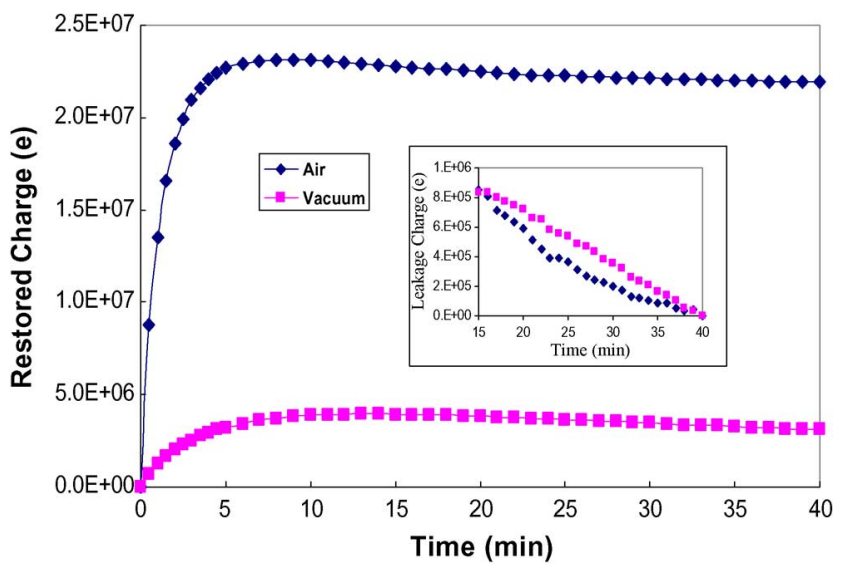

Fig. 11. Measured restored charge as a function of time in air and in vacuum after $-5 \mathrm{~V}$ to $4 \mathrm{~V}$ voltage step applied to test capacitor.

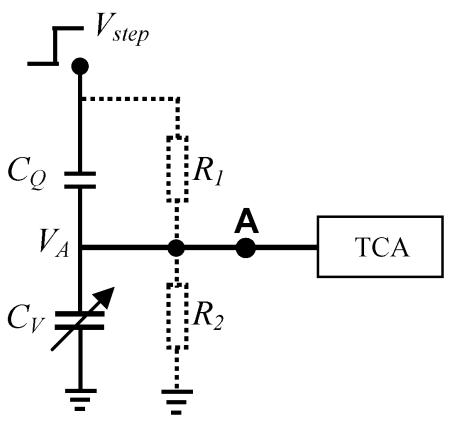

Fig. 12. Stability model if electrometer.

to the extremely small amounts of charge to be measured. For example, $1 \mathrm{~V}$ applied on a capacitor with a resistance of $10^{13} \Omega$ produces a leakage current of $0.1 \mathrm{pA}$, sufficiently large and not to be ignored in a circuit model. A model of the electrometer which includes resistors to model leakage paths is shown in Fig. 12, wherein the capacitor resistance acts as a voltage divider. Upon applying a voltage step, the dc voltage at node A shifts to an equilibrium value set by the ratio of the resistors $R_{1}$ and $R_{2}$. The time required for this shift is determined by the associated RC time constant. $R_{1}$ corresponds to the leakage through the air gap on-chip capacitor, and $R_{2}$ is the oxide resistance to ground. In vacuum, the electrical resistance of the gap is larger than gap resistance in air, so the equilibrium dc rest voltage in vacuum is possibly smaller than that in air. This is illustrated in Fig. 11 by the difference in the restored charge observed in both cases.

The inset of Fig. 11 shows that after the restored charge reaches an initial peak, it then falls gently, indicating some sort of charge leakage occurring. The leakage currents in air and vacuum cases are similar, which is approximately $88.7 \mathrm{aA}$. The source of leakage is very likely to be due to parasitic dielectric charging in the buried oxide layer and the native oxide on the structure [15]. The $\mathrm{SiO}_{2}$ provides trap sites for positive and negative charges both deep in the volume and at the interfaces between multilayer stacks. Applying a voltage on the anchors therefore generates parasitic charges on the $\mathrm{SiO}_{2}$, resulting in leakage, occurring over long time spans. Further investigation is underway to ascertain the contribution of other parasitic leadage sources. 


\section{CONCLUSION}

The design, modeling, and experimental results for a novel micromachined charge sensor were discussed in this paper. To reduce the quiescent charge leakage at the input node, a novel setup with an electrically isolated capacitor was introduced between the variable capacitor and input to sensor electronics. Methods to improve the sensitivity and resolution are suggested while the long-term stability of these sensors is modeled and discussed.

The isolation capacitor used in this paper is an off-chip capacitor, which induces more than $2 \mathrm{pF}$ parasitic capacitance and limits the sensitivity. The provision of an on-chip integrated air-gap capacitor $C_{1}$ offers a practical method to increase the sensitivity, by reducing parasitic capacitor $C_{p a}$ in (6). The demonstrated high sensitivity of this device has also been used to probe and investigate naturally occurring charge inducing phenomena, otherwise unobservable apart from the high precision capability discussed herein.

\section{ACKNOWLEDGMENT}

The authors thank I. Haneef for wire bonding the devices presented in this paper.

\section{REFERENCES}

[1] F. Krueger and J. Larson, "Chipmunk IV: Development of and experience with a new generation of radiation area monitors for accelerator applications," Nucl. Instrum. Meth. Phys. Res. A, vol. 495, pp. 20-28, 2002.

[2] C. I. Calle, J. G. Mantovani, C. R. Buhler, E. E. Groop, M. G. Buehler, and A. Nowicki, "Embedded electrostatic sensors for Mars exploration missions," J. Electrostatics, vol. 61, pp. 245-257, 2004.

[3] E. R. Badman and R. G. Cooks, "Miniature mass analyzers," J. Mass Spectrom., vol. 35, pp. 659-671, 2000.

[4] G. Zimmerli, T. M. Giles, R. L. Kautz, and J. M. Martinis, "Noise in the Coulomb blockade electrometer," Appl. Phys. Lett., vol. 61, pp. 237-239, Jul. 1992.

[5] A. N. Cleland and M. L. Roukes, "A nanometer-scale mechanical electrometer," Nature, vol. 392, pp. 160-162, Mar. 1998.

[6] H. Kroemmer, A. Erbe, A. Tilke, S. Manus, and R. H. Blick, "Nanomechanical resonators operating as charge detectors in the nonlinear regime," Europhys. Lett., vol. 50, pp. 101-106, Apr. 2000.

[7] H. Palevsky, R. K. Swank, and R. Grenchik, "Design of dynamic condenser electrometers," Rev. Sci. Instrum., vol. 18, pp. 298-314, 1947.

[8] G. Rietveld and H. E. Brom, "Vibration reed electrometer for accurate measurement of electrical currents below $10 \mathrm{pA}$," IEEE Trans. Instrum. Meas., vol. 56, no. 2, pp. 559-563, Apr. 2007.

[9] P. S. Riehl, K. L. Scott, R. S. Muller, R. T. Howe, and J. A. Yasaitis, "Electrostatic charge and field sensors based on micromechanical resonators," J. Microelectromech. Syst., vol. 12, pp. 577-589, Oct. 2003.

[10] P. S. Riehl, K. L. Scott, R. S. Muller, and R. T. Howe, "High-resolution electrometer with micromechanical variable capacitor solidstate sensor," in Proc. Actuator and Microsystem Workshop, 2002, pp. 305-308.
[11] J. E.-Y. Lee, Y. Zhu, and A. A. Seshia, "Room temperature electrometry with SUB-10 electron charge resolution," J. Micromech. Microeng., vol. 18, p. 025033, Feb. 2008.

[12] [Online]. Available: http://www.memscap.com/en_mumps.html

[13] Y. Zhu, J. Lee, and A. A. Seshia, "MEMS electrometer system simulation using a time-domain variable capacitor model," in Proc. 14th IEEE Transducers, Lyon, France, Jun. 2007, pp. 1685-1688.

[14] J. Lee, Y. Zhu, and A. A. Seshia, "A variable capacitor based MEMS electrometer," in Proc. Eurosensors XX, Sep. 2006, pp. 452-453.

[15] J. Wibbeler, G. Pfeifer, and M. Hietschold, "Parasitic charging of dielectric surfaces in capacitive microelectromechanical systems (MEMS)," Sens. Actuators A, vol. 71, no. 1-2, pp. 74-80, Nov. 1998.

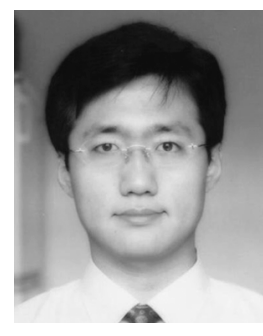

Yong Zhu received the B.Sc. degree in physics and the M.Eng. degree in microelectronics from Dalian University of Technology, Dalian, China, in 1999 and 2002, respectively, and the Ph.D degree in microelectronics from the Peking University, Beijing, China, in 2005.

He was a Research Associate with the Department of Engineering, University of Cambridge, Cambridge, U.K., in 2006 and 2007 with the project of microfabricated electrometer and mass sensor. $\mathrm{He}$ is currently a Research Academic in the School of Electrical Engineering and Computer Science, University of Newcastle, Australia. His research interests include microfabrication, micromachined sensors, design of interface circuits for MEMS, and modeling of MEMS.

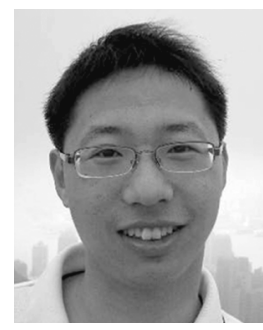

Joshua E.-Y. Lee (S'05) received the M.Eng. and B.A. degrees in electrical engineering from Cambridge University, Cambridge, U.K., in 2005. He is currently pursuing the Ph.D. degree in the Division of Mechanics, Materials and Design, Department of Engineering, Cambridge University.

His research interests include micromechanical resonators for the measurement of mass and charge, plus timing applications, as well as analog circuit design for MEMS.

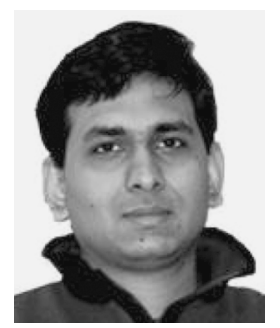

Ashwin A. Seshia (S'98-M'02) received the B.Tech. degree in engineering physics from the Indian Institute of Technology (IIT), Bombay, India, in 1996, and the M.S. and Ph.D. degrees in electrical engineering and computer sciences from the University of California, Berkeley, in 1999 and 2002, respectively.

$\mathrm{He}$ is currently a member of the Faculty of the Department of Engineering, University of Cambridge, Cambridge, U.K., where he is a Lecturer in microelectromechanical systems (MEMS), a Fellow of Queens' College, and a member of the Micromechanics and Nanoscience research groups. His research interests include integrated micromechanical vibratory structures for sensor and timing applications, micromachined devices for in vivo monitoring, and biological sensor systems. 